\title{
The Scheme to Optimize the Throughput of the Inspection System
}

\author{
Haoran Sun \\ North China Electric Power University, Baoding 071000, China \\ Corresponding author e-mail: 2858773155@qq.com
}

Keywords: Airport Security Check, Process Optimization, Monte Carlo Simulation

\begin{abstract}
In this paper, we study a scheme to optimize the throughput of the inspection system. Firstly, the checkpoint sequence model based on control sequence is established, which serves as the basis of the latter model. Secondly, the channel quantity optimization model based on queuing theory is established to optimize the throughput of the inspection system. A series of equations are set up to explore the flow of passengers through a security checkpoint. Next, Monte Carlo algorithm is used to simulate queuing process. An optimization function is established with the aim of minimizing the total cost which contains the waiting cost and the security inspection cost.
\end{abstract}

\section{Introduction}

In recent years, the frequency of travel is increasing with the continuous improvement of people's living standards. At the same time, people are more willing to choose the plane as their travel tool. However, frequent passenger blockage appears in the airport area, which leads to airport safety issues and passenger dissatisfaction and so on. This paper establishes two models for this problem, which are the checkpoint sequence model and the channel quantity optimization model based on queuing theory, aiming at exploring the solution to this problem to optimize the airport throughput

\section{The Check Point Sequence Model}

The Check point Sequence System. The check point sequence system is based on the Daganzo control of sequence system [1]checkpoint sequence system diagram is shown in Figure1.

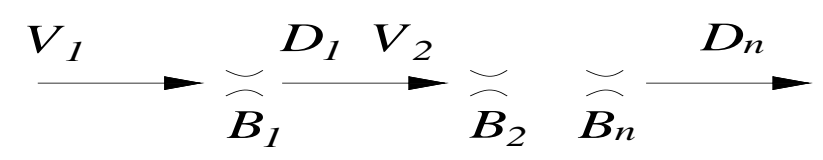

Fig.1Schematic diagram for sequence bottleneck system

In the figure, $B_{i}$ represents the i-th grade checkpoint; $V_{i}$ represents the input of the i-th grade checkpoint; $D_{i}$ represents the output of the i-th grade checkpoint.

The system satisfies the following theorem:[1]

Theorem 1: The output of $B_{i-1}$ is equal to the input of $B_{i}$, if the travel time between $B_{i}$ and $B_{i-1}$ is a fixed value.

Theorem 2: The discharge capacity of the whole system is consistent with the one with the least capacity.

Checkpoint Sequence System of Airport Security Inspection. The bottleneck of the airport security system is ID check, security check and baggage collection. The simplified process is shown as below.:

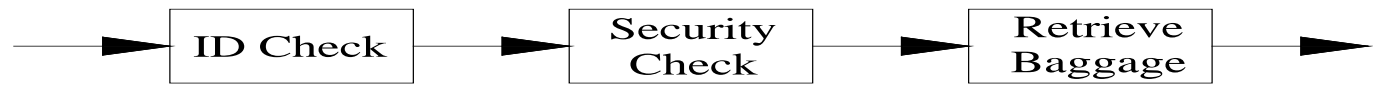

Fig.2 Time series of airport security system

In order to optimize the airport security inspection system, due to the complexity of the security system structure, it is impossible to optimize the entire system by individually optimizing a checker in the system. In order to illustrate this problem, we establish the airport system input and output curves, as shown in Figure 3. [1] 


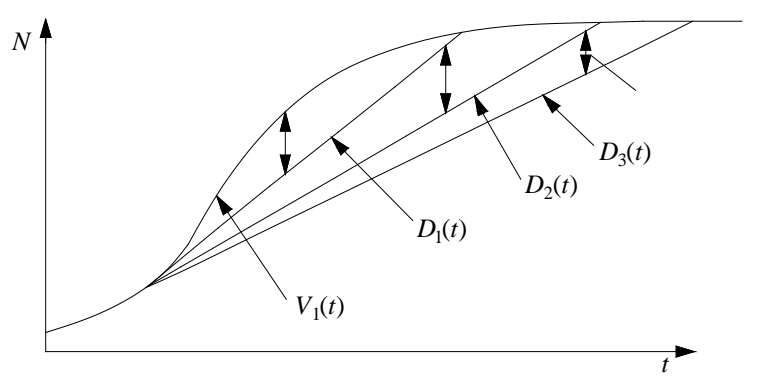

Fig.3 Input- output graph of airport bottleneck system

In the Fig3, the $\mathrm{x}$-axis represents time, while the $\mathrm{y}$-axis represents the cumulative number of passengers arriving. The curve $\mathrm{V}_{1}(\mathrm{t})$ represents the cumulative number of the passengers at ID check, $D_{1}(\mathrm{t}), D_{2}(\mathrm{t}), D_{3}(\mathrm{t})$ respectively represents the number of passengers leaving ID check, security check and retrieving baggage. The curve $\mathrm{V}_{1}(\mathrm{t})$ is time-depended. The area enclosed by the adjacent function curves is denoted by $S$ as the passenger waiting time in the ticket vending machine, security equipment and automatic ticket gate machine, which is delay. In order to reduce delays, to reduce the delay of a single bottleneck may lead to the transfer of bottlenecks, the system does not reduce the delay so the total delay to consider $S$ which is calculated by Equation (1).

$$
S=S_{1}+S_{2}+S_{3}
$$

Where, $S$ is the total delay; $S_{\mathrm{i}}$ is the delay of each segment

\section{The Channel Quantity Optimization Model}

Model Preparation. (1)The passenger arrival distribution follows the Poisson distribution with parameter

(2)The service time of the channel is subject to a negative exponential distribution with the parameter $\mu$.

(3)Travelers follow the first-come-first-served (FCFS) queuing rule.

(4)Passengers will not abandon service because of long queues.

(5)Passengers are not allowed to change after entering the queue.

Model Establishment. When the whole queue arrives at steady state, we can get:[2-4]

$$
\begin{gathered}
\rho=\frac{\lambda}{C \mu}<1 \\
p_{0}(c)=\left[\sum_{k=0}^{c-1} \frac{1}{k !}\left(\frac{\lambda}{\mu}\right)^{k}+\frac{1}{c !} \frac{1}{(1-\rho)}\left(\frac{\lambda}{\mu}\right)^{k}\right]^{-1} \\
p_{n}(C)= \begin{cases}\frac{1}{n}\left(\frac{\lambda}{\mu}\right) p_{0}(C) \quad n=1,2, \cdots, C \\
\frac{1}{C ! C^{n-c}}\left(\frac{\lambda}{\mu}\right) p_{0}(C) \quad n=C+1\end{cases}
\end{gathered}
$$

Where, $\lambda$ is the average arrival rate of passengers; $\mu$ denotes the average service rate; $\rho$ is the service intensity; $p_{n}(C)$ is the probability of $\mathrm{n}$ passengers in the system at any time in the $C$ channels.

When the system reaches the equilibrium state, the average waiting time $T$ of each customer in the system is:

$$
E(T)=\frac{p_{n}(C)}{C \mu(1-\rho)^{2}}=\frac{n \mu}{n !(n \mu-\lambda)^{2}}\left(\frac{\lambda}{\mu}\right)^{n} p_{0}(C)
$$

The length of queue $L_{s}$ is calculated by Equation(6):

$$
L_{S}=L_{q}+C \rho=\frac{1}{C !} \frac{(C \rho)^{C} \rho}{(1-\rho)^{2}} P_{0}+\frac{\lambda}{\mu}
$$

In the queuing system, the passengers want more channels, less waiting time, so that their time to minimize the cost of loss, for which the airline will increase the number of channels to meet the needs 
of passengers, However, Airlines can’t input inspection equipment without limitation. Optimization design is needed. The passenger waiting time is converted into the waiting cost, and the following optimization target model is established with the objective of minimizing the total cost of the passenger waiting cost and the increased equipment service fee.

Our objective function can be represented as:

$$
\min Z(C)=C_{s} C+C_{w} L_{s}
$$

Where, Cs is the cost per unit time required for each channel; $C w$ denotes the queuing costs for each passenger in the system; $Z(C)$ is the total cost; $C *$ is the point that minimizes the objective function $Z(C)$, and $C *$ satisfies:

$$
\begin{gathered}
\left\{\begin{array}{l}
Z\left(C^{*}\right) \leq Z\left(C^{*}-1\right) \\
Z\left(C^{*}\right) \leq Z\left(C^{*}+1\right)
\end{array}\right. \\
L_{s}\left(C^{*}\right)-L_{s}\left(C^{*}+1\right) \leq \frac{C_{S}}{C_{w}} \leq L_{s}\left(C^{*}-1\right)-L_{s}\left(C^{*}\right)
\end{gathered}
$$

\section{The Process of Solving the Model}

According to the actual monitoring data at an airport in America ,the average arrival rate of passengers $\lambda$ is 0.077 , the average service rate $\mu$ is 0.08 . What's more, $C_{S}$ is $26.3, C_{w}$ is $47.2[1]$.

The Monte Carlo stochastic simulation method is used to solve the model. The concrete process is as follows:[5]

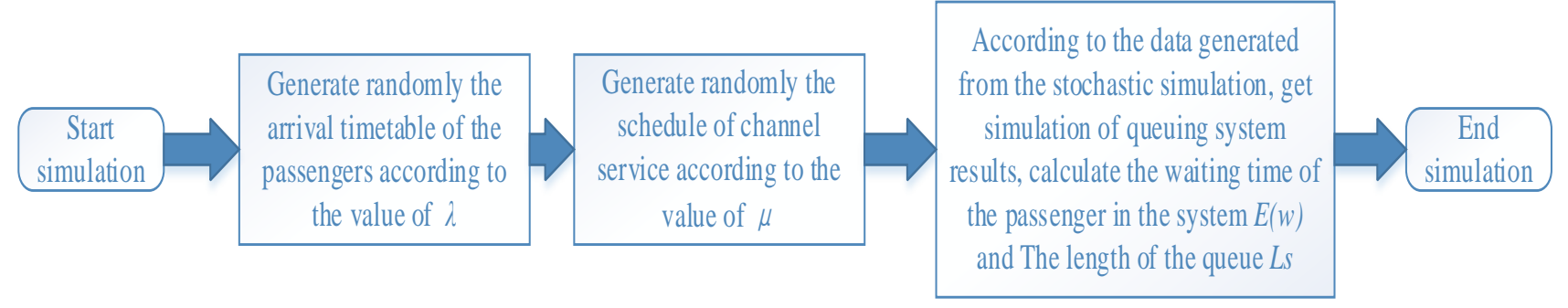

Fig.4 Flow chart for Monte Carlo simulation process

Simulation of the waiting time of the passengers and queue length changes as follows:
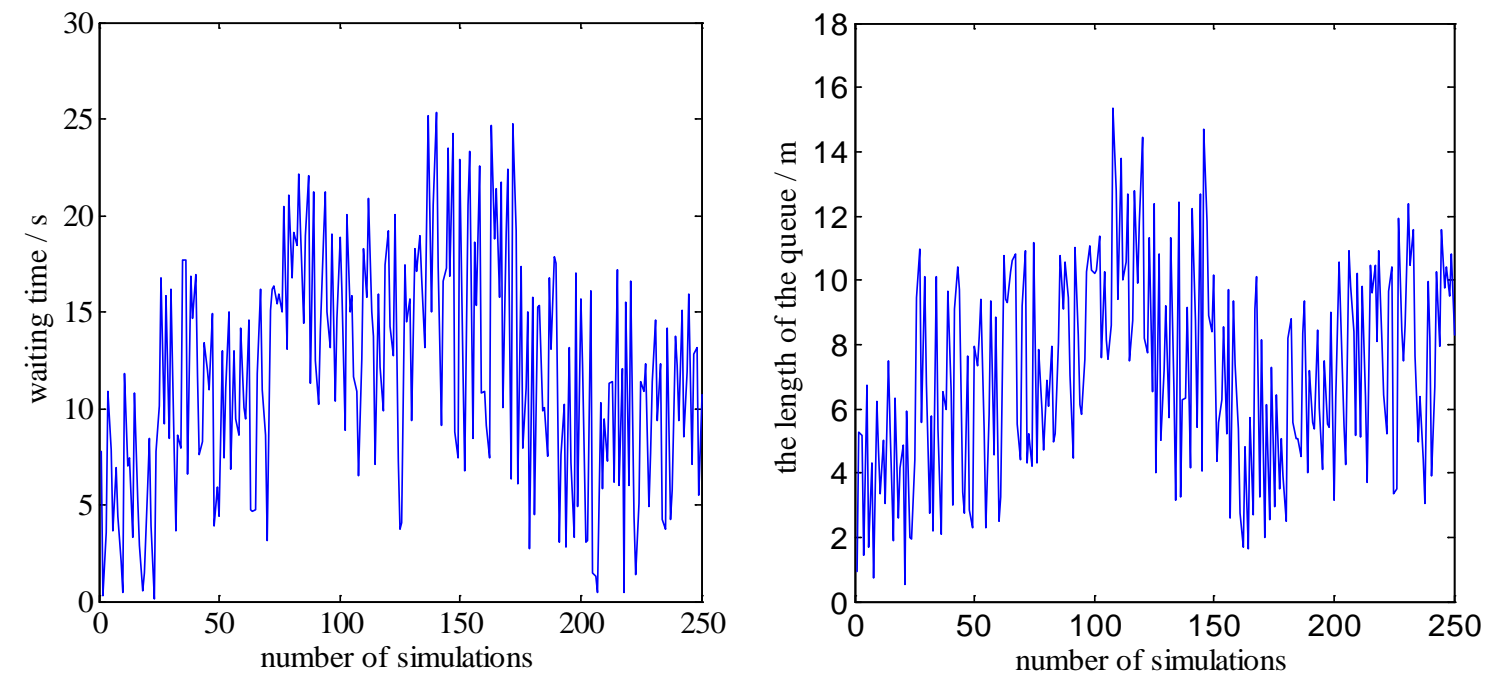

Fig.5 The results of Monte Carlo

After the Monte Carlo simulation is complete, we put the simulation results into the model, and can get the optimization results as follow. From the table above, we know that only the number of channels $C^{*}=4$ satisfies the Eq.(5), as a result, the optimal number of channels is 4 . 
Table.5Dfferential value of the length of queue

\begin{tabular}{|c|c|c|c|}
\hline$L_{S}(1)-L_{S}(2)$ & -2.8147 & $L_{S}(4)-L_{S}(5)$ & -1.9153 \\
\hline$L_{S}(2)-L_{S}(3)$ & -1.7926 & $L_{S}(5)-L_{S}(6)$ & 0.0249 \\
\hline$L_{S}(3)-L_{S}(4)$ & 1.3223 & $L_{S}(6)-L_{S}(7)$ & 0.0007 \\
\hline
\end{tabular}

\section{Conclusion}

In this paper, two models are established to solve the problem of airport passenger congestion. We find the best method to calculate the number of channels by the establishment of the model. Applying the actual observation data for an airport to this model, we get the best number of channels by the method of Monte Carlo random number simulation, which also proves the correctness and practicability of this model.

\section{References}

[1] Jie Liu, Shengxue He, Haodong Zhang, et al, "Optimization Analysis on Sequence Bottleneck System of Urban Rail Transit,” Computer Application,January2016, Pages 271-274+286.

[2]Guanghui Xu, “Stochastic Service System,” The Science Publishing Company,1980.

[3] Rongfang Shen, “Operational Research,” Machinery Industry Press.

[4]Jie Zhang, Shuo Zhou, "the Model of Operational Research,” Northeastern University Press, 2005.

[5] Yaowu Jiang, "Study on the Quantity Configuration of Ticketing and Security Checking Equipment for High - speed Railway Stations,” Central South University, 2013. 